\title{
Meta
}

Journal des traducteurs

Translators' Journal

\section{Cinq notions de télédétection aérospatiale : un exemple de structuration d'un champ terminologique}

\section{Loïc Depecker}

Volume 34, numéro 2, juin 1989

URI : https://id.erudit.org/iderudit/002537ar

DOI : https://doi.org/10.7202/002537ar

Aller au sommaire du numéro

Éditeur(s)

Les Presses de l'Université de Montréal

ISSN

0026-0452 (imprimé)

1492-1421 (numérique)

Découvrir la revue

Citer cet article

Depecker, L. (1989). Cinq notions de télédétection aérospatiale : un exemple de structuration d'un champ terminologique. Meta, 34(2), 280-284.

https://doi.org/10.7202/002537ar

\section{Résumé de l'article}

Le point de départ de la structuration du champ terminologique est le problème posé par le terme angloaméricain ground control point dans le domaine de la télédétection aérospatiale. Le calque courant point de contrôle est en fait l'équivalent français avec point de vérification, de checkpoint. Ce mauvais usage du terme point de contrôle incite à définir rigoureusement les concepts français auxquels correspond ground control point: point d'appui et point de canevas, termes correspondant à deux notions distinctes par lesquelles ground control point se traduit en français; point de repère et amer qui entrent dans la sphère de ces notions; et enfin point de vérification et son synonyme correct point de contrôle. Ces cinq notions sont analysées ici comme constituant un champ terminologique. 


\section{ÉTUDES TERMINOLOGIQUES ET LINGUISTIQUES}

\section{CINQ NOTIONS DE TÉLÉDÉTECTION AÉROSPATIALE : UN EXEMPLE DE STRUCTURATION D'UN CHAMP TERMINOLOGIQUE}

Le point de départ de la recherhce ${ }^{1}$ est la mauvaise traduction fréquemment donnée à ground control point en télédétection aérospatiale à savoir point de contrôle. Le syntagme angloaméricain fait en lui-même difficulté, le terme anglais control étant comme chaque fois ambigu : veut-il dire commande, conduite, contrôle 2 ? De plus, point de contrôle est connu en français depuis plus d'un siècle dans le domaine de la photogrammétrie $^{3}$ : c'est le synonyme de point de vérification, notion utilisée pour apprécier les résultats d'un traitement géométrique des données. Et leur équivalent angloaméricain est pour l'un et l'autre check point. D'où une double démarche: une fois dénoncé le calque point de contrôle pour ground control point, il s'agit de rechercher un équivalent français adéquat pour ground control point; et de montrer ensuite quels sont la signification et le statut de point de contrôle dans l'ensemble des notions qui lui correspondent en télédétection aérospatiale.

L'équivalent point d'appui est avancé à titre d'essai pour ground control point avec la définition suivante: «Point matérialisé au sol par un détail naturel ou artificiel, connu en coordonnées et identifiable sur l'image ${ }^{4}$ ». La définition paraît claire à première vue mais l'on constate que de point d'appui est également proche une autre notion: le point de canevas (en angloaméricain ground control point précisément). Notion elle aussi connue depuis longtemps en photogrammétrie, le point de canevas désigne un point du sol localisé par un détail naturel ou artificiel identifiable sur un cliché et connu en coordonnées. En fait cette décomposition de la notion de point de canevas se trouve précisément synthétisée par la définition qui a servi à définir précédemment point d'appui.

Il est alors décidé de substituer pour cette définition point de canevas à point d'appui. En effet, ce dernier est tout d'abord un point de canevas. La différenciation qui s'effectue ainsi est importante: outre la hiérarchisation de chacune des deux notions le point de canevas est plus générique que le point d'appui - il y a émergence d'un autre concept, point d'appui, à partir de l'analyse faite sur point de canevas dont la notion paraît alors stable: le compte rendu de la séance du 6 octobre 1966 mentionné précédemment est formel sur ce sujet: «point d'appui : la définition est entièrement reprise et développée à partir de celle de point de canevas ${ }^{5}$ ». Le point d'appui est alors défini comme un «point de canevas utilisé pour effectuer le prétraitement et le traitement géométrique des données ${ }^{\%}$.

Mais c'est à ce moment que les éléments nouvellement définis, point de canevas, et point d'appui, remettent en cause les deux autres notions appartenant à la zone du savoir dans lequel ils s'inscrivent: point de repère notamment, et amer. Pour ce qui est de point de repère, sa signification s'impose très vite comme une évidence, et l'acception qu'il a en langue commune en donne déjà clairement le sens: c'est un «point matérialisé au sol par un détail naturel ou artificiel identifiable sur l'image 7 ». La modification qui intervient par rapport au sens que point de repère a en langue commune se situe dans le fait qu'il entre dans le champ d'un domaine de spécialité, la télédétection aérospatiale. 
Et dans ce domaine l'image est un des principaux éléments de référence: c'est elle qui est traitée et interprétée. Ainsi la définition de point de repère mentionne-t-elle l'image de façon significative. Puis à nouveau, la structuration que la langue anglaise effectue avec landmark (équivalent courant de point de repère) et seamark prolonge la réflexion : il convient alors de traiter également seamark, - en français amer - de façon à vérifier notamment si aucune contradiction n'apparaît à l'examen conjoint des notions de seamark et de landmark. Pour amer, la définition vient assez rapidement : «Repère terrestre ou repère marin visible destiné à la navigation maritime ou aérienne ${ }^{8}$ ». L'emprise du domaine aérospatial n'est pas totalement agissante ici. Mais l'intérêt de ce traitement repose moins dans la définition que dans la note qui accompagne le traitement d'amer: «Il est incorrect d'employer le terme amer pour désigner un point d'appui». On remarque en effet dans quelques textes cette extension de sens qui est d'autant plus un abus qu'amer a un sens utile et indiscutable. C'est précisément le recours au concept de point d'appui précédemment délimité qui peut mettre fin à l'erreur qui consisterait à prendre l'un pour l'autre. Mais dans un domaine aussi précis, il convenait de s'interroger sur une telle erreur: si amer subissait une distorsion de sens, c'est que les chercheurs avaient l'intuition d'une notion nouvelle, mais sans parvenir ou penser à la définir ni conceptuellement, ni terminologiquement: il faut en effet pour que les concepts s'inscrivent dans un savoir donné, qu'ils aient une représentation linguistique qui les rende sensibles mais qui, surtout, leur soit conforme.

Pour finir, une dernière notion est proposée: point de vérification dont l'équivalent angloaméricain courant est check point. Son intégration aux notions précédemment traitées, particulièrement point de canevas et point d'appui, intéresse en premier lieu le travail d'adéquation entre le repérage géographique d'un point et sa localisation sur une image. La première définition s'élabore à partir de point de canevas mais est différentielle par rapport à celle de point d'appui. Point de vérification: «Point de canevas autre qu'un point d'appui ${ }^{9}{ }$... Ensuite, la référence à point d'appui est gommée au profit de la définition suivante: «Point de canevas utilisé pour apprécier les résultats d'un prétraitement ou d'un traitement géométrique des données». Mais ce libellé efface le rapport au point d'appui, ce qui peut encore une fois prêter à confusion. Il est donc décidé de revenir, lors de la séance plénière de la commission du 4 décembre 1986 , à la définition distinctive précédente: «Point de vérification: Point de canevas autre que le point d'appui, utiliser pour apprécier les résultats d'un prétraitement et/ou d'un traitement géométrique des données».

On constate l'étroite parenté des trois notions: point de canevas, qui domine conceptuellement les deux autres, point d'appui et point de vérification, elle-même la résultante des deux précédentes. Par rapport à ces trois notions, la plus proche demeure point de repère, et plus lointaine mais non moins importante, amer. On arrive alors à la fiche de travail de la séance du 4 décembre assortie des modifications de dernière lecture: «Point de vérification»: point de canevas autre qu'un point d'appui $10_{\gg}$...: on passe du défini à l'indéfini, la généralité de la définition ne visant pas un cas de figure particulier. Pour point de canevas, la définition se contracte, grâce au profit qui est tiré du traitement rigoureux des notions connexes: «Point de repère connu en coordonnées et identifiable sur l'image. À amer enfin la mention voir aussi point de repère est ajoutée pour souligner le rapport qu'amer entretient avec cette notion: la première ne concerne pas le repérage sur une image alors que c'est le cas pour la seconde. C'est pourquoi il est refusé, de façon concomitante, d'ajouter dans la définition «Point de repère terrestre ou marin...» On aboutit ainsi à trois notions hiérarchisées: point de canevas, point d'appui et point de vérification; avec une notion connexe: point de repère qui ne 
concerne sur l'image que le repérage lui-même. Et une notion proche: amer, qui n'entre pas dans le champ des quatre autres mais leur est nécessaire pour les fixer définitivement dans leur clarté.

Les cinq notions se présentent donc à l'issue du traitement terminologique qui leur est donné dans l'ordre logique suivant:

1. Point de repère (n.m.) a.a.: landmark

Définition: Point matérialisé au sol par un détail naturel ou artificiel, identifiable sur l'image.

V.a.: amer, point de canevas.

2. Point de canevas (n.m.) a.a.: (ground, picture) control point

Définition: *Point de repère connu en coordonnées.

Note 1: Les coordonnées peuvent être connues en planimétrie et/ou en altimétrie.

Note 2: La traduction de «control point» par «point de contrôle» est incorrecte.

V.a.: amer, point d'appui, point de vérification.

3. Point d'appui (n.m.) a.a.: ground control point

Définition: *Point de canevas utilisé pour effectuer le prétraitement et/ou le traitement géométriques des données.

V.a.: donnée brute, donnée corrigée, donnée élaborée, donnée prétraitée, imagette, prétraitement d'image.

4. Point de vérification (n.m.) a.a.: check point

Synonyme: point de contrôle, n.m.

Définition: *Point de canevas, autre qu'un *point d'appui, utilisé pour apprécier les résultats d'un prétraitement et/ou d'un traitement géométrique des données.

Note: L'équivalent point de contrôle, donné également à check point, est exact, mais son emploi doit être déconseillé par suite de la confusion qu'induit l'angloaméricain check point.

5. Amer (n.m.)

a.a.: seamark

Définition: Repère terrestre ou marin visible destiné à la navigation maritime ou aérienne.

Note: Il est incorrect d'employer le terme amer pour désigner un *point d'appui, un *point de canevas ou un *point de vérification.

V.a.: point de repère.

En conclusion, il est possible de faire les constatations suivantes :

c'est l'angloaméricain, notamment sous sa forme ground control point qui a servi de vecteur à la recherche; mais ce vecteur, pris isolément, reste inopérant: faisant irruption à titre d'emprunt dans la langue des spécialistes français, son sens est fluctuant et son rapport à leur savoir indéfini; le rôle de l'angloaméricain se trouve également mis en valeur par les parallélismes morphologiques qu'il induit (exemple seamark/landmark) ce qui permet d'effectuer de proche en proche des vérifications à mesure que la structuration des concepts s'élabore en français;

cette structuration des concepts en français ne s'effectue pas moins de façon autonome, ce que montre par exemple la création de la notion bien délimitée de point d'appui par rapport à point de canevas ; 
ce mouvement de va-et-vient entre les deux langues est fondamental mais se fait ici au profit du français qui s'appuie sur les notions souvent vagues de l'angloaméricain pour préciser, structurer et rendre opératoire un savoir bien délimité ;

ce travail de structuration met en valeur un aspect souvent négligé de la traduction, pourtant très utile aux chercheurs : c'est en traduisant la langue de l'autre qu'on structure et transcende son propre savoir.

Enfin on peut considérer comme un champ terminologique les cinq notions définies ci-dessus en soulignant l'intérêt qu'il peut y avoir à utiliser en terminologie, notamment pour l'élaboration de corpus, les concepts de notions hiérarchisées, notions connexes, et notions proches.

LOIC DEPECKER

Conseiller technique au Commissariat général de la langue française (France)

\section{REMERCIEMENTS}

Au Professeur Serge Paul pour ses conseils, et aux membres de la commission ministérielle de terminologie de la télédétection aérospatiale.

\section{RÉSUMÉ}

Le point de départ de la structuration du champ terminologique est le problème posé par le terme angloaméricain ground control point dans le domaine de la télédétection aérospatiale. Le calque courant point de contrôle est en fait l'équivalent français avec point de vérification, de check point. Ce mauvais usage du terme point de contrôle incite à définir rigoureusement les concepts français auxquels correspond ground control point : point d'appui et point de canevas, termes correspondant à deux notions distinctes par lesquelles ground control point se traduit en français; point de repère et amer qui entrent dans la sphère de ces notions; et enfin point de vérification et son synonyme correct point de contrôle. Ces cinq notions sont analysées ici comme constituant un champ terminologique.

\section{ACKNOWLEDGMENTS}

I am grateful to Professor Serge Paul for his numerous advice and to the members of the internal working group of the departmental terminology committee for remote sensing.

ABSTRACT

The terminological research begins with the translation into French of the term ground control point in the remote sensing field. Actually the usual calque point de contrôle is the French equivalent, along with point de vérification, of check point. The improper use of point de contrôle leads to explain accurately the French concepts to which ground control point refers. The terms point d'appui and point de canevas correspond to two different notions by which ground control point is translated into French: point de repère and amer which belong to the sphere of these notions; finally point de vérification and its exact synonym point de contrôle. These five notions are studied as parts of a terminological field.

Notes

1. Cette question a été abordée et traitée par la commission ministérielle de terminologie de la télédétection aérospatiale (COMITAS) au cours de l'année 1986.

Cette instance, rattachée à la direction générale des enseignements supérieurs et de la recherche du ministère français de l'Éducation nationale, fut créée par l'arrêté du 20 avril 1978 (Journal officiel N.C. du 6 mai 1978). 
L'exposé qui suit est une synthèse et un résumé des débats auxquels a donné lieu la recherche de l'équivalent français exact de ground control point.

2. Voir sur ce sujet l'article de Pierre Daviault paru dans le numéro 60 de la revue Traduire.

3. La photogrammétrie est l'ensemble des théories, techniques et équipements qui permettent de déterminer la forme, les dimensions et éventuellement la position dans l'espace d'objets à partir de photographies de ces objets (Jean Cruzet in la Banque des mots, 1976, $\mathrm{n}^{\circ} 11, \mathrm{pp} .71-92$ ).

4. Dossier proposé à la séance plénière de la COMITAS le 21 octobre 1986, p. 6 (diffusion interne).

5. P. 5 du procès-verbal susmentionné.

6. Notes de séance prises lors de la réunion plénière de la COMITAS du 2 octobre 1986.

7. P. 7 du procès-verbal susmentionné.

8. P. 7 du procès-verbal susmentionné.

9. Notes de séance prises lors de la réunion plénière du 2 octobre 1986.

10. Notes de séance. 